\title{
Flux lattice imaging of a patterned niobium thin film
}

\author{
M. Roseman ${ }^{\text {a) }}$ and P. Grütter \\ Department of Physics, Centre for the Physics of Materials, McGill University, Montréal, \\ Québec H3A 2T8, Canada
}

A. Badía

Departmento Física de la Materia Condensada, University of Zaragoza, Zaragoza, Spain

V. Metlushko

Department of Electrical Engineering and Computer Science, University of Illinois at Chicago, Chicago, Illinois 60607

Using our cryogenic magnetic force microscope, we have investigated a superconducting $\mathrm{Nb}$ thin film, $100 \mathrm{~nm}$ in thickness with $T_{c} \sim 6.5 \mathrm{~K}$. The film is patterned with a square array $(1 \mu \mathrm{m}$ $\times 1 \mu \mathrm{m})$ of antidots, which serve as artificial pinning centers for magnetic flux. We have observed flux lattice matching as a function of applied magnetic field and temperature, for field strengths up to the third matching field, with evidence of flux dragging by the tip around the antidots. Force gradient distance curves acquired at temperatures about $T_{c}$ clearly demonstrate an observable Meissner force between tip and sample, and allow for an estimation of the magnetic screening penetration depth. (C) 2001 American Institute of Physics. [DOI: 10.1063/1.1355327]

\section{INTRODUCTION}

Interest in the interplay between artificial pinning centers on patterned superconducting films, and the flux-line lattice, particularly at "matching fields," has produced numerous studies with the aim of understanding and controlling flux pinning and flow. ${ }^{1-4}$ Many of these employ bulk measurements, such as magnetization and electrical resistivity, which do not address the question of the particular spatial arrangement of fluxoids, an issue still not well understood. By contrast, the magnetic force microscope is ideally suited for this task, producing three dimensional magnetic images which allow for the direct visualization of flux-line lattice position. ${ }^{5}$

In this article, we present an overview of the design of our cryogenic magnetic force microscope, a noise analysis of the system, as well as some results obtained on a patterned superconducting $\mathrm{Nb}$ thin film.

\section{MICROSCOPE DESIGN}

The microscope, constructed almost exclusively using stainless steel, is approximately $10 \mathrm{~cm}$ long and $5 \mathrm{~cm}$ in diameter, allowing for its insertion into the $3 \mathrm{in}$. diam bore of an $8 \mathrm{~T}$ superconducting magnet. Feedthroughs into the vacuum can allow for electrical connections, an optical fiber, and pumping lines for a turbomolecular vacuum pump. Cooling is achieved with the aid of He exchange gas, which is pumped out prior to imaging. Vibration isolation is achieved through suspending the microscope below a soft, nested-type stainless steel bellows, which attenuates accelerations by more than an order of magnitude over a $200 \mathrm{~Hz}$ bandwidth.

The optical fiber forms part of the fiber-optic based interferometer system, ${ }^{6}$ used to detect cantilever deflections. This sensor is particularly well suited for low temperture

\footnotetext{
a) Author to whom correspondence should be addressed; electronic mail: roseman@physics.mcgill.ca
}

systems, as only one end of the fiber need be subjected to cryogenic temperatures, while other electronic components remain at room temperature.

In situ movement of both the optical fiber and sample with respect to the fixed cantilever is achieved using a piezoelectric based positioner. ${ }^{7}$ Step sizes of $10-20 \mathrm{~nm}$ for the optical fiber positioner, measured using interferometric techniques, are routinely achieved at $4.2 \mathrm{~K}$.

Cantilevers used for magnetic imaging are made magnetically sensitive by evaporating a magnetic coating on the tip. During imaging, the cantilever is excited at its resonance frequency by a small piezoelectric actuator. As the cantilever is scanned with respect to the sample, changes in the resonance frequency (indicative of the local force gradient sensed by the cantilever) are tracked by a phase-locked loop (PLL). ${ }^{8}$ The PLL then provides a dc signal proportional to the cantilever resonance frequency, which serves as a feedback signal upon which constant force-gradient images are acquired. Scan ranges of 40,14 , and $8 \mu \mathrm{m}$ are achieved at room temperature, $77 \mathrm{~K}$, and $4.2 \mathrm{~K}$, respectively.

Temperature measurement and control using a GaAlAs diode and Lake Shore Cryotronics 320 proportional integral differential temperature controller system allows for the sample temperature to be varied from 4.2 to $15 \mathrm{~K}$ when operating in a liquid helium environment. Small applied magnetic field strengths, up to 100 Gauss, are measured using an in situ flux gate magnetometer.

\section{NOISE CHARACTERISTICS}

Of equal importance to its capabilities is an appreciation of the microscopes' limitations. Such information aids not only in the optimization of the apparatus, but also serves as a guide for selecting suitable experiments. To this end, we have undertaken an analysis of our system, with particular emphasis placed on the noise contributions, both electronic and mechanical in origin. 


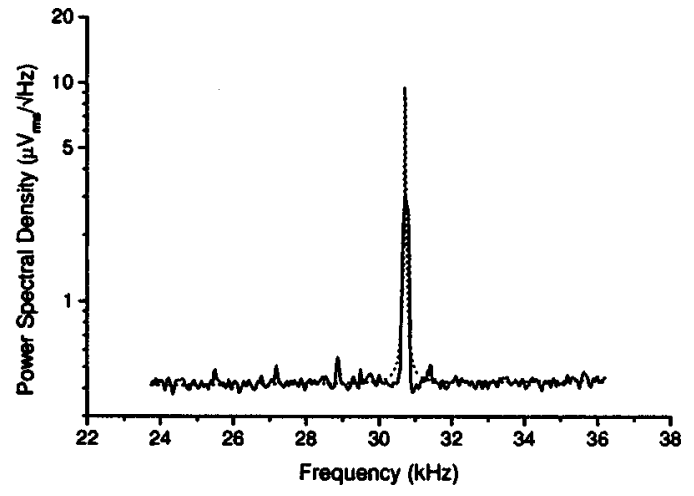

FIG. 1. Measured (solid line) and calculated (dotted line) power spectral density of the deflection signal from a thermally driven silicon nitride cantilever at $5 \mathrm{~K}$.

This study involves, in part, modeling various noise contributions, including laser shot, intensity, phase and optical feedback noise, thermal cantilever motion, as well as Johnson noise, gain-peaking effects, and other terms associated with the deflection sensor (current-voltage converter) electronics. Results of this analysis are shown in Fig. 1, which illustrates both calculated and measured power spectral density plots of the deflection signal from a thermally driven silicon nitride cantilever at $5 \mathrm{~K}$.

Often, the sensitivity of a force microscope is correlated to the observability of the thermally driven cantilever resonance (peak in Fig. 1). The uniform background noise level is however, highly relevant in determining the achievable frequency resolution while operating in ac mode. This can easily be seen by transforming the spectrum of Fig. 1 into the time domain. The background noise results in a jitter of the zero crossings of the sinusoidal oscillations at the cantilever resonant frequency, thereby limiting the frequency resolution. ${ }^{8-10}$ It is thus the signal to noise ratio (the signal at the peak, and the noise of the background) that determines the ultimate sensitivity.

A more complete analysis of noise contributions ${ }^{9}$ suggests that shot noise of the interferometer dominates the background noise in our system. Recently we have achieved a frequency resolution of $0.34 \mathrm{~Hz}$ in a $72 \mathrm{~Hz}$ bandwidth, corresponding to a force gradient resolution of approximately $2.2 \times 10^{-6} \mathrm{~N} / \mathrm{m}$.

\section{RESULTS}

The sample is a superconducting $\mathrm{Nb}$ film, $100 \mathrm{~nm}$ in thickness, patterned with a square lattice of antidots, of period $1 \mu \mathrm{m}$ and diameter $0.3 \mu \mathrm{m}$, which serve as artificial pinning centers for magnetic flux. It was produced using laser interferometric lithography, and exhibits a $T_{c} \sim 6.5 \mathrm{~K}$. One of $\mathrm{us}^{3}$ has investigated the magnetoresistivity, magnetization, and critical current behavior of this sample as a function of "matching field," so named because at these values the flux lattice spacing becomes commensurate with the lattice of antidots. This results in a majority of the magnetic field becoming trapped as quantized fluxoids within the antidots.
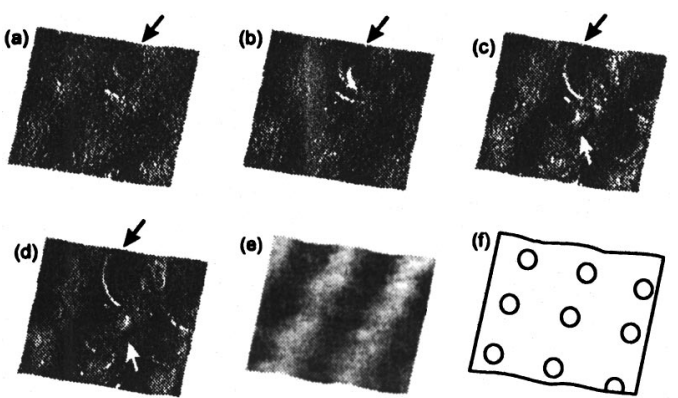

FIG. 2. Panels (a)-(d) are constant height images, acquired at successively smaller tip-sample distances. The height interval between panels is $75 \mathrm{~nm}$, with (a) the farthest and (d) the closest to the surface. Panel (e) is an AFM image and (f) a drawing of the region, which reveals the position of the antidots. All are $2 \mu \mathrm{m} \times 2 \mu \mathrm{m}$ in area. Measurements were made at a temperature of $5.5 \mathrm{~K}$ in a field of $62.1 \mathrm{G}$ (the third matching field). Tip induced movement of the flux around the antidots (indicated by arrows) is evident for smaller tip-sample separations. The asymmetry of the structures is attributed to the left-to-right motion of the cantilever during image acquisition.

Imaging was performed under various conditions of temperature and applied field. As shown in Fig. 2, strong evidence of flux matching is observed, with each flux line situated adjacent to an antidot. Interactions between the stray field of the tip and the flux lines, particularly at smaller tipsample separations, produce significant flux motion, apparent as crescent shaped structures surrounding the antidot.
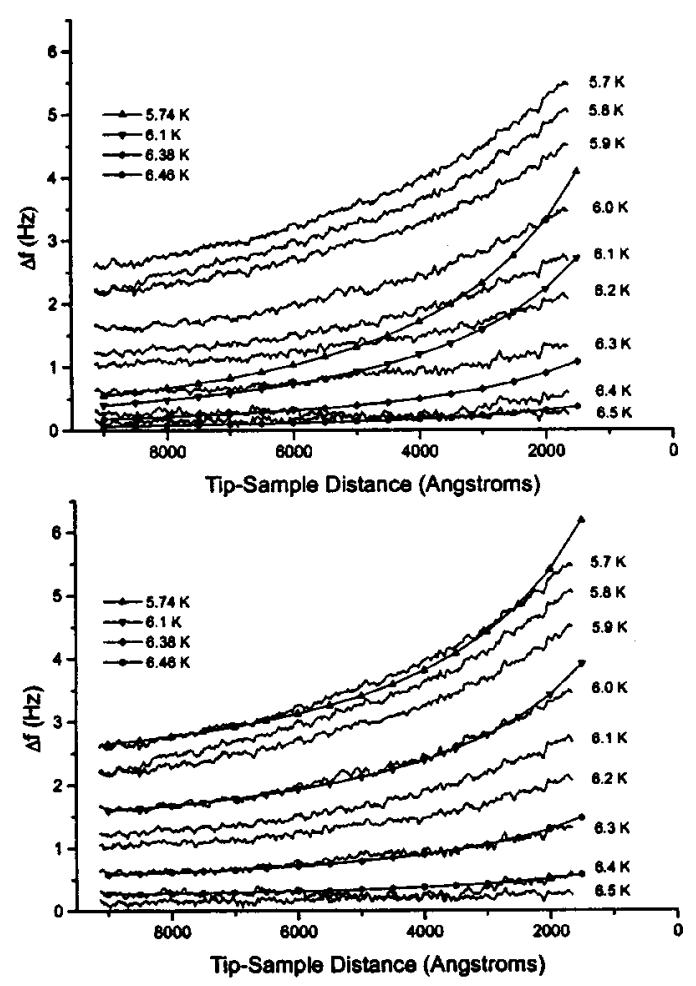

FIG. 3. Experimental and simulated approach curves. The upper graph shows the original data sets, while in the lower graph the vertical position of the theoretical curves has been adjusted to emphasize overlap between the theoretical and experimental data. Temperature discrepancies between corresponding curves of the two data sets are within the experimental uncertainty of our temperature sensor. 
As the magnetic tip is approached toward the superconducting sample, the repulsive Meissner force produces an increase in the resonance frequency of the cantilever. Theoretically, this response can be modeled as follows. The vertical magnetic force between the superconductor and an extended tip a distance $a$ above, may be written as ${ }^{11}$

$$
\begin{aligned}
F_{z}(a)= & \frac{\mu_{0}}{4 \pi} \int_{0}^{\infty} d k \int_{V} d^{3} \mathbf{r} \int_{V} d^{3} \mathbf{r}^{\prime}\left\{k^{3} C_{2_{x y}}(k) e^{-2 a k} e^{-k\left(z+z^{\prime}\right)}\right. \\
& \left.\times\left[\sum_{i, j} M_{i}(\mathbf{r}) g_{i j}\left(\mathbf{r}-\mathbf{r}^{\prime}\right) M_{j}\left(\mathbf{r}^{\prime}\right)\right]\right\}
\end{aligned}
$$

where $\mathbf{M}(\mathbf{r})$ is used for the tip magnetization function and $V$ for the volume of the tip. $g_{i j}$ is a certain metric tensor which arises by writing the field equation solutions in cylindrical coordinates, and $C_{2_{x y}}$ is a coupling coefficient involving the penetration depth, film thickness, and a wave number. For the case of a pyramidal tip and a thin film coating of magnetic material, uniformly magnetized along the axis Eq. (1) specializes to

$$
\begin{aligned}
F_{z}(a)= & \frac{\mu_{0} M^{2} t^{2}}{4 \pi} \int_{0}^{\infty} d k \int_{-c}^{c} d y \int_{-c}^{c} d y^{\prime} \int_{|y| h / c}^{h} d z \int_{|y| h / c}^{h} d z^{\prime} \\
& \times k^{3} C_{2_{x y}}(k) e^{-2 a k} e^{-k\left(z+z^{\prime}\right)} J_{0}\left[k\left(y-y^{\prime}\right)\right],
\end{aligned}
$$

where $h$ and $c$, respectively, stand for the tip's height and half width, $t$ for the coating thickness, and $J_{0}$ is the zeroeth order Bessel function of the first kind.

A series of approach curves exhibiting the Meissner effect are shown in Fig. 3, which illustrate both simulated and experimental data acquired as a function of temperature under zero magnetic field. We attribute the mismatch between theoretical and experimental data (upper graph) as arising from difficulties in the modeling of the tip. This is consistent with the fact that other tip geometries (e.g., cylindrical and conical) produce qualitatively similar curves, although the actual value of the frequency shift depends strongly on the tip shape. In addition, the effect of the antidot lattice has been neglected. The theoretical curves in the lower graph in Fig. 3 have had their vertical positions adjusted, and show good agreement with experimental values. From this work we estimate the magnetic screening length $\lambda_{0} \simeq 150 \mathrm{~nm}$, which agrees fairly well with previously reported values. ${ }^{12}$

\section{CONCLUSION}

We have designed and built a cryogenic magnetic force microscope, capable of operation within a temperature range of $4.2 \mathrm{~K}$ to room temperature, in magnetic fields of up to $8 \mathrm{~T}$. A thorough analysis of the noise sources present in our system shows that the current frequency resolution of $0.34 \mathrm{~Hz}$ in $72 \mathrm{~Hz}$ bandwidth is limited by the shot noise of the interferometer. We have used this system to study a superconducting $\mathrm{Nb}$ thin film, patterned with an array of antidots. Magnetic images and approach curves were acquired, as a function of applied magnetic field and temperature. An analysis of our results yields an estimate of the magnetic screening length, $\lambda_{0} \simeq 150 \mathrm{~nm}$.

\section{ACKNOWLEDGMENTS}

The authors would like to thank P. LeBlanc for the evaporation of the magnetic tips. The work by M. Roseman and P. Grütter was supported by grants from the National Science and Engineering Research Council of Canada and Le Fonds pour la Formation de Chercheurs et l'Aide à la Recherche de la Province de Québec.

${ }^{1}$ M. Baert, V. V. Metlushko, R. Jonckheere, V. V. Moshchalkov, and Y. Bruynseraede, Phys. Rev. Lett. 74, 3269 (1995).

2 J. I. Martin, M. Velez, J. Nogues, and I. K. Schuller, Phys. Rev. Lett. 79, 1929 (1997).

${ }^{3}$ V. Metlushko, U. Welp, G. W. Crabtree, R. Osgood, S. D. Bader, L. E. DeLong, Z. Zhang, S. R. J. Brueck, B. Ilic, K. Chung, and P. J. Hesketh, Phys. Rev. B 60, 12585 (1999).

${ }^{4}$ A. Terentiev, B. Watkins, L. E. DeLong, L. D. Cooley, D. J. Morgan, and J. B. Ketterson, Physica C 332, 5 (2000)

${ }^{5}$ A. Moser, H. J. Hug, B. Stiefel, and H. J. Güntherodt, J. Magn. Magn. Mater. 190, 114 (1998), and references therein.

${ }^{6}$ D. Rugar, H. J. Mamin, and P. Güthner, Appl. Phys. Lett. 55, 2588 (1989).

${ }^{7}$ S. H. Pan, Piezo-electric motor, international Patent publication No. WO93/19494, International Bureau, World Intellectual Property Organization (30 September 1993).

${ }^{8}$ U. Dürig, H. R. Steinauer, and N. Blanc, J. Appl. Phys. 82, 3641 (1997).

${ }^{9}$ M. Roseman and P. Grütter, Rev. Sci. Instrum. 71, 3782 (2000).

${ }^{10}$ Y. Liu, Ph.D. thesis, McGill University, 1997.

${ }^{11}$ A. Badia, Phys. Rev. B 60, 10436 (1999); 63, 094502 (2001).

${ }^{12}$ H. Zhang, J. W. Lynn, C. F. Majkrzak, S. K. Satija, J. H. Kang, and X. D. Wu, Phys. Rev. B 52, 10395 (1995). 